\title{
Evaluating service performance for clinical governance
}

\author{
Gill Wakley
}

\section{Introduction}

The standards of service delivery should be those that allow you to practise as a competent clinician. You may be competent but be unable to perform or practise to a competent level if the resources available to you are inadequate, or other colleagues have insufficient knowledge or skills to support you. You cannot be expected to take all the responsibility for ensuring that the resources you need to be able to practise in a competent manner are available. However, as a professional you should play a significant role in collecting evidence to make a case for the need for essential resources for your staff or colleagues to the Trust or primary care organisation, to colleagues or managers, or whomever is appropriate.

Many of the methods used to establish individual performance are relevant for evaluating service performance. ${ }^{1}$ Defining what your service provides gives you a yardstick against which to measure your service performance. Use several methods to identify overlaps or gaps in your service development or delivery and the learning needs of your organisation, so that you validate the findings of one method by another. No one method will give you reliable information. Colleagues from different disciplines could usefully comment on any shortfalls or overlaps in how your work interfaces with theirs. Patients or people who do not use your services could tell you whether the way you operate or provide services is off-putting or inappropriate. There may be data about the performance or outcomes of your service that could point out those gaps in your service provision of which you were previously unaware.

\section{Assessment by an external body}

Patients and local people are asked to comment on their experiences of local health services to the Healthcare Commission (the new name for the Commission for Health Improvement since April 2004). The review team talks to staff, other local health care organisations and organisations that represent patients and the public. The review focuses on the Trust's or primary care organisation's arrangements for clinical governance. These include the condition of premises, the ability of the leaders, how well the organisation manages potential risks to patients, and what opportunities it gives to staff to improve their skills. People, including patients and staff, can give their views by letter, e-mail or telephone. They can talk to a member of the review team in a confidential one-to-one meeting. The Healthcare Commission publishes its findings in a report that highlights areas of good practice and areas that need to be improved. ${ }^{2}$

\section{Internal assessment}

Internal assessment should typically involve patients and the public. Patient and public involvement may occur at three levels:

J Fam Plann Reprod Health Care 2005; 31(2): 136-138

Staffordshire University, Stafford, UK

Gill Wakley, MD, MFFP, Visiting Professor in Primary Care

Development and Freelance General Practitioner

Correspondence to: Professor Gill Wakley.

E-mail: gillwak@aol.com
- Asking individual patients about their own care.

- Asking about the range and quality of health services on offer.

- Planning and organising health service developments.

If a patient involvement or public consultation exercise is to be worthwhile, it has to involve representatives from the section of the population that you want to know about. Set up systems to seek out and involve people from minority groups or those with sensory impairments such as blind or deaf people. ${ }^{3}$

Write down and consult on what you are intending to do, define the purpose of the exercise, select an appropriate method depending on your target population and your resources, and obtain the commitment of everyone who will be affected. You might hold focus groups, or set up a patient panel, or invite feedback and help from a patient participation group. You could interview patients selected either at random from the patient population or chosen for their experience of a particular condition or circumstance.

\section{Audit}

Adopt the six aspects of the National Health Service (NHS) performance assessment framework: ${ }^{4}$ health improvement, fair access, effective delivery, efficiency, patient and carer experience, and health outcomes. Keep your evaluation as simple as possible and avoid wasting resources on unnecessarily bureaucratic evaluation. The extent to which you can evaluate your service will depend on the quality of your records and extent to which you use the capacity of computers.

Structural audits might concern resources such as equipment, premises, skills, people, and so on. Process audits focus on what is done to the patient; for instance, clinical protocols and guidelines. Audits of outcomes consider the impact of care or services on the patient and might include patient satisfaction, health gains, and effectiveness of care or services. You might look at aspects of quality of the structure, process and outcome of the delivery of the clinical field in which you work - focusing on access, equity of care between different groups in the population, efficiency, economy, effectiveness for individual patients, or other areas..$^{5}$ Set standards for your performance, find out how you are doing, search to find out best practice, make the changes and then re-audit the care given to patients in the future with the same problem.

\section{Compare the systems in your service with those} required by legislation

Legislation changes quite frequently. You could start by comparing the systems in your practice or organisation with those required by the Disability Discrimination $\mathrm{Act}^{6}$ and Health and Safety legislation. ${ }^{7}$

\section{Compare performance against guidelines}

You might find out about the standards and learning needs of the organisation by collecting all the protocols or guidelines that are used in your service and rationalising them so that you have one common set. Working as a team you can compare your knowledge and usual practice with others and with protocols or guidelines. ${ }^{8,9}$ Audit to find out how often people adhere to such a protocol or guideline, and if they can justify why they deviate from the recommendations. 


\section{Assess risk}

Risk assessment entails evaluating the risks to the health, well-being or competence of yourself, staff and/or patients in your practice or workplace, and deciding on the action needed to minimise or eliminate those risks. ${ }^{10,11}$ Be clear about the difference between:

- Harm: what occurs if a risk is realised and an adverse event has occurred.

- Risk: the potential that harm may occur.

Spend time wisely by only evaluating risks that are large, high-volume, have caused concern or are expensive. Risks may be prevented, avoided, minimised or managed where they cannot be eliminated. You, your colleagues and your staff may need to learn how to do this.

As part of continually identifying service development needs, record significant events where someone has experienced an adverse event or had a near miss. Most significant incidents do not have one cause. Usually there are faults in the system, which are compounded by someone or several people being careless, tired, overworked or poorly informed. Cultivate an atmosphere of openness and discussion without blame so that you can all learn from the significant event. If people think they will be blamed, they will hide the incident and no one will be able to prevent it happening again. Look for all the causes and try to remedy as many as possible to prevent the situation from arising in the future.

\section{Health needs assessment}

A health needs assessment is a balance of what should be done with what can be done and what can be afforded..$^{12}$ It has three components:

- A description of the health problems of a population.

- Identifying inequalities in health and access to services.

- Determining priorities for the most effective use of resources.

A detailed account of how you might carry out a sexual health needs assessment appears in Improving Sexual Health Advice. ${ }^{13}$ Without a sexual health needs assessment you cannot know whether you are providing the services that are required, or just the ones that have grown up over the years.

\section{Review teamwork}

Measure the effectiveness of the team in which you work ${ }^{14}$ by evaluating whether the team has:

- Clear goals and objectives

- Accountability and authority

- Individual roles for members

- Shared tasks

- Regular internal formal and informal communication

- Full participation by members

- Confrontation of conflict

- Feedback to individuals

- Feedback about team performance

- Outside recognition

- Two-way external communication

- Team rewards.

\section{Service development needs}

Look for service development needs reflecting why patients receive a poor quality of service such as:

- Inadequately trained staff or staff with poor levels of competence.

- Lack of confidentiality.

- Staff not being trained in the management of emergency situations.

- Doctors or nurses not being contactable in an emergency or being ineffective.
- Treatment being unavailable due to poor management of resources or services.

- Insufficient numbers of available staff for the workload.

- Qualifications of locums or deputising staff being unknown or inadequate for the posts they are filling.

- Arrangements for transfer of information from one team member to another being inadequate.

- Team members not acting on information received.

Many of these items will need action as a team, but for some of them it may be your responsibility to ensure that adequate standards are met. Some of these service needs will highlight training needs.

\section{Training needs analysis}

Identify the range and extent of training needs from service needs (i.e. expectations in the sexual health strategy and local service delivery strategies). Next, specify the training needs very precisely. An organisation could do this by pooling the results after the workforce has completed selfassessment questionnaires. Feedback from peers, patients and managers adds other information about the training needs of the individuals. Finally, analyse how best the training needs might be met. 15

\section{Reflect on whether you are providing cost-effective} care and services

Cost-effectiveness is not synonymous with 'cheap'. A costeffective intervention is one which gives a better or equivalent benefit from the intervention in question for lower or equivalent cost, or where the relative improvement in outcome is higher than the relative difference in cost. Being cost effective means having the best outcomes for the least input. Using the term 'cost effective' implies that you have considered potential alternatives.

An intervention must first be considered clinically effective to warrant investigation into its potential to be cost effective. Evidence-based practice must incorporate clinical judgement. You have to interpret the evidence when it comes to applying it to individual patients, whether it is evidence about clinical effectiveness or costeffectiveness. A new or alternative treatment or intervention should be compared directly with the previous best treatment or intervention.

An economic evaluation is a comparative analysis of two or more alternatives in terms of their costs and consequences. There are four different types:

1. Cost effectiveness analysis is used to compare the effectiveness of two interventions with the same treatment objectives.

2. Cost minimisation compares the costs of alternative treatments that have identical health outcomes.

3. Cost utility analysis enables the effects of alternative interventions to be measured against a combination of life expectancy and quality of life; common outcome measures are quality-adjusted life years or healthrelated quality of life.

4. Cost-benefit analysis is a technique designed to determine the feasibility of a project, plan, management or treatment by quantifying its costs and benefits. It is often difficult to determine these accurately with regard to health.

While health valuation is unavoidable, it cannot be objective. You will probably have learning needs around what subjective method it is best to use. ${ }^{16}$

Efficiency is sometimes confused with effectiveness. Being efficient means obtaining the most quality from the least expenditure, or the required level of quality for the least expenditure. To measure efficiency you need to 
make a judgement about the level of quality of the 'purchase' and be able to relate it to 'price'. 'Price' alone does not measure efficiency. Quality is the indicator used in combination with price to assess if something is more efficient. So, cost-effectiveness is a measure of efficiency and suggests that costs have been related to effectiveness.

\section{Set priorities}

Consider if you have service development needs. Discuss whether:

- The current skill mix in your team is appropriate.

- More cost-effective alternative types of delivery of care are available.

- Sufficient staff training exists for those taking on new roles and responsibilities.

Group and summarise your service development and learning needs from the exercises you have carried out. Grade them according to the priority you set. You may put one at a higher priority because it fits in with those established from another section, or put another lower because it does not fit in with other activities needed. Collect information from all the team, the patients, users and carers to feed back before you make a decision on how to progress. Remember to take external influences into account such as governmental priorities, the content of the Local Delivery Plan, and so on

Select those topics that are tied into organisational priorities, have clear aims and objectives, and are achievable within your time and resource constraints. When ranking topics for action or learning in order of priority consider whether:

- The project aims and objectives are clearly defined.

- The topic is important (a) for the population served (e.g. the size of the problem and/or its severity) and (b) for the skills, knowledge or attitudes of the individual or team.

- It is feasible.
- It is affordable.

- It will make enough difference.

- It fits in with other priorities.

You will still have more ideas than can possibly be implemented. Remember the highest priority - the health service is for patients that use it or who will do so in the future.

Statements on funding and competing interests

Funding. None identified.

Competing interests. None identified.

References

1 Wakley G. Evaluating individual performance for clinical governance. J Fam Plann Reprod Health Care 2005; 31: 46-48. http://www.healthcarecommission.org uk.

3 Chambers R, Drinkwater $\mathrm{C}$, Boath E. Involving Patients and the Public: How to do it Better (2nd edn). Oxford, UK: Radcliffe Publishing, 2003

4 http://www.dh.gov.uk/assetRoot/04/05/71/84/04057184.pdf

5 Chambers R, Wakley G. Clinical Audit in Primary Care: Demonstrating Quality and Outcomes. Oxford, UK: Radcliffe Publishing, 2005

6 http://www.hmso.gov.uk/acts/acts1995/95050--b.htm.

7 http://www.hsedirect.com

8 Department of Health. Effective Commissioning of Sexual Health and HIV Services - A Sexual Health and HIV Commissioning Toolkit for Primary Care Trusts and Local Authorities. London, UK: Department of Health, 2003

9 http://www.ffprhc.org.uk.

10 Mohanna K, Chambers R. Risk Matters in Healthcare. Oxford, UK: Radcliffe Publishing, 2000.

11 National Patient Safety Agency (NPSA). Introduction to 7 Steps for Patient Safety. 2004. http://www.npsa.nhs.uk.

12 Wright J. Health Needs Assessment in Practice. London, UK: BMJ Books, 1998.

13 Wakley G, Cunnion M, Chambers R. Improving Sexual Health Advice. Oxford, UK: Radcliffe Publishing, 2003.

14 Chambers R, Wakley G. Making Clinical Governance Work for You. Oxford, UK: Radcliffe Publishing, 2000.

15 Bee F, Bee R Training Needs Analysis and Evaluation. London, UK: Institute of Personnel and Development, 1997.

16 McCulloch D. Valuing Health in Practice. Aldershot, UK: Ashgate Publishing Ltd, 2003.

\section{Website Review}

\section{WOW: WellBeing of Women}

This website states that it exists to fund vital research into reproductive health. It quotes: "Our mission is to put an end to fear and suffering from women's reproductive problems".

WellBeing of Women (WOW) raises funds through events, campaigns, corporate partnerships, collaborative activities with likepartnerships, collaborative activities with like-
minded organisations, and an extensive network of branches and supporters across the UK. The website contains pages where you can donate or complete gift aid to the charity. A list of the projects funded is just a click away. The research projects have a strong UK element, but are international. For example, they recently funded research into vulval lichen sclerosis and also into the relationship between chlamydia, ectopic pregnancy and infertility. The research funded by WOW falls into three categories of investigation:

- gynaecological cancers

- pregnancy and birth

- quality of life problems such as polycystic ovary syndrome, endometriosis, troublesome symptoms of the menopause, painful periods and incontinence.

They will soon be adding sexual health as an area in which more research needs to be done. The subjects that they are interested in supporting are often identified by surveys so that they are those important to women. I was surprised to read that so many women find menstruation such a bad experience, but less surprised by some of the other concerns such as ignorance about sexually transmitted infections (STIs).

Health professionals are too late to apply for funding support for this year, but you might want to start thinking about research funding for 2006 onwards. The charity also allocates funding for research training fellowships.

A 24-page copy of the magazine celebrating their 40th anniversary takes a little time to load as it contains many pictures. It includes information about surveys, research and events.

A section on reproductive health information lists various useful leaflets on some common problems: polycystic ovaries, cystitis, postnatal depression, prematurity, hysterectomy, menopause, ovarian cancer, pregnancy and birth. These can be purchased by post (the website gives the bulk-buy rates) or downloaded. Contact information for various organisations related to these subjects appears in this section.

My two caveats about the usefulness of this website and of the charity was the lack of information on contraception and STIs and the apparent emphasis on middle-class, Western worries about reproductive health. The first area is, of course, due to my bias - what I think is important! The second area is probably due to marketing considerations. People are more likely to give money to something they think may benefit them or people they know - not the poor, the disadvantaged or problems in the developing countries. Clearly the charity does support research in these areas but the website material came across (to me) as a little parochial.

Recommend this site to well-heeled friends, think about joining in the support, and bear WOW in mind if you need research funds. Website: http://www.wellbeingofwomen.org. uk.

Reviewed by Gill Wakley, MD, MFFP Visiting Professor in Primary Care Development, Staffordshire University and Freelance GP, Writer and Lecturer, Abergavenny, UK

\section{Visit the Faculty Website at: www.ffprhc.org.uk}

